\section{Madres y padres de adolescentes urbanos: percepciones y expectativas de la atención en salud sexual y reproductiva para sus hijos}

\author{
MARÍA XIMENA LUENGO-CHARATH ${ }^{1,2, a}$, \\ TERESA MILLÁN-KLÜSSE ${ }^{3, \mathrm{~b}}$, JOSEFINA HERREROS A., ${ }^{4, \mathrm{c}}$, \\ ANA J. ZEPEDA O. ${ }^{2,5, \mathrm{~d}}$, MARÍA EUGENIA HENRÍQUEZ C. ${ }^{6}$
}

\section{Parental perceptions and expectations about sexual and reproductive health care for urban adolescents}

Studies have reported which expectations and demands adolescents have for more accessible sexual and reproductive health (SRH) services. However, there is limited information regarding parents' requirements and demands for SRH services their adolescents need. Aim: To explore the knowledge of parents regarding $S R H$ needs of their adolescent daughters and sons and what they demand from primary health care services (PHCS). Material and Methods: A quali-quantitative, descriptive, cross-sectional study was performed. Nine fathers and 24 mothers were interviewed to design a survey. In the quantitative phase, 125 mothers voluntarily, anonymously and confidentially were interviewed. All participants were users of PHCS of urban communities in the Metropolitan Area showing a high percentage of births by adolescents' mothers. Data analysis was descriptive and analytical. Results: Interviews served as input to design the questionnaire. Seventy seven percent $(77,6 \%)$ of interviewed mothers knew that their sons/daughters, regardless of their gender, had concern about sexuality; their main fear was adolescent pregnancy. Seventy six percent (76\%) was aware that their sons/daughters might request attention in SRH clinics and 97\% agreed that they should require these services during early adolescence. The most desirable features of such services were identified. Conclusions: Parents recognize that they would prefer their sons/daughters postponed sexual initiation; however, they are aware that pregnancy prevention is needed. Mothers approve SRH services. They agree that counseling to prevent pregnancies and sexual transmitted diseases (STD) and AIDS was an important issue. They require support for themselves and their sons/daughters.

(Rev Med Chile 2014; 142: 1259-1266)

Key words: Adolescent; Fathers; Mothers; Parents; Reproductive healthPrimary Health Care.

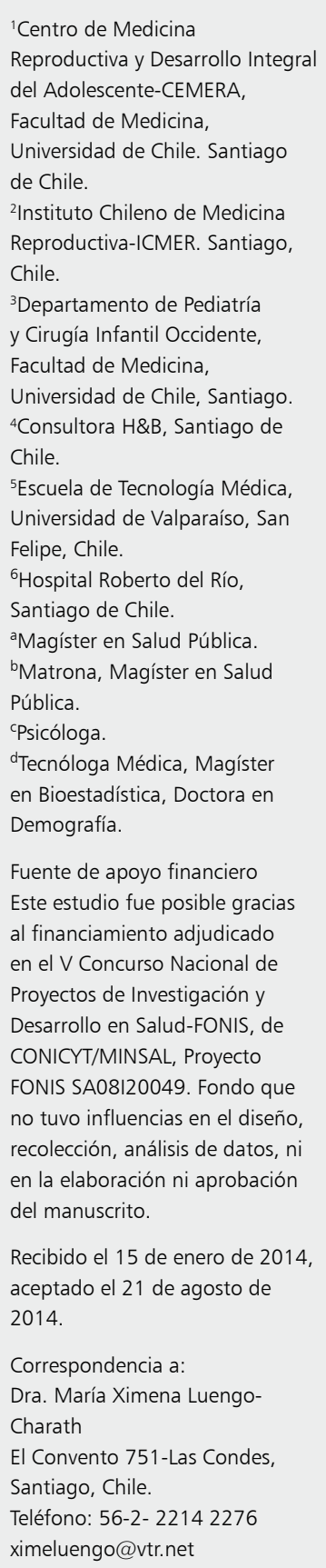

T mportantes avances en salud pública orientada a adolescentes ha habido en Chile, destacando el Programa y la Política Nacional de Salud para Adolescentes y Jóvenes, con sus respectivas orientaciones $^{1-4}$. Recomendaciones técnicas de agencias internacionales para servicios amigables $y$ de calidad son conocidas, así como publicaciones sobre opiniones y demandas de los adolescentes $^{5-9}$. Sin embargo, persisten barreras de acceso a atención de salud sexual y reproductiva (SSR) 
para adolescentes ${ }^{2,10,11}$. Publicaciones señalan que los adolescentes siguen necesitando ayuda para prevenir embarazo y VIH/Sida ${ }^{13-17}$. En Chile, disminuir el embarazo adolescente es una meta de los Objetivos de Desarrollo del Milenio ${ }^{18}$.

Es importante conocer la visión de madres y padres de adolescentes, actores claves para el desarrollo saludable de sus hijos. El presente estudio exploró conocimientos y opiniones de usuarios del sistema público de salud, sobre las necesidades de SSR de sus hijos/as adolescentes e identificó expectativas de atención, para contribuir a implementar políticas y programas. Se presentan principalmente resultados cuantitativos y una síntesis de hallazgos cualitativos previos.

\section{Sujetos y Método}

Estudio exploratorio-descriptivo, de corte transversal, con etapas cualitativa y cuantitativa, realizado el año 2009. El estudio se realizó en servicios de salud (SS) metropolitanos, que presentaban las tasas más altas de nacidos vivos de madres adolescentes el 2005: Norte (SSMN), Occidente (SSMOc) y Sur Oriente (SSMSO). El objetivo de la primera etapa era recoger información para diseñar una encuesta dirigida a madres.

\section{Etapa cualitativa (madres y padres)}

Entrevistas en profundidad a 24 madres, proporcionales para cada uno de los SS seleccionados y estrato de edad de los hijos/as, incluyendo también a madres de usuarias de centros especializados para adolescentes (CEA). Los 9 padres de adolescentes usuarios del sistema público de salud entrevistados, pertenecían a la Región Metropolitana, sin distinción por SS.

El análisis de contenido se realizó en matrices con las categorías definidas y segmentadas según sexo de los padres, tramo etario de los hijos y pertenencia a atención primaria de salud (APS) o CEA.

\section{Etapa cuantitativa (madres)}

El diseño de muestreo fue bietápico (consultorio/madre) y estratificado por comuna. El tamaño muestral fue calculado en 125 madres, el cual permite estimar un intervalo de confianza de 95\% para el indicador: "proporción de madres que saben, conocen o intuyen que sus hijos/as pueden solicitar atención en los servicios públicos de SSR", cuya hipótesis fue $80 \%$. La precisión de la estimación sería de 7 puntos porcentuales.

La distribución de la muestra fue proporcional a la población adolescente de cada SS: contemplando 2, 3 y 3 consultorios y 27, 42 y 56 encuestas, para los SSMN, SSMOc y SSMSO, respectivamente.

Toda madre perteneciente a la población objetivo presente el día de la encuesta, contactada en salas de espera, podía participar. La participación fue voluntaria, anónima y confidencial, previo consentimiento informado aprobado por el Comité de Ética independiente asesor de ICMER. Sólo 3 madres rechazaron participar $(2,3 \%)$.

La encuesta fue evaluada por jueces expertos y se realizó prueba piloto. El análisis de los datos fue descriptivo y analítico, con medidas de tendencia central y de frecuencia. Además, se estratificó para el análisis de resultados según quinquenios de edad ( 10 a 14 y 15 a 19) y sexo de los hijos adolescentes. Los datos se procesaron en el programa estadístico SPSS.

\section{Resultados}

\section{Fase cualitativa: madres}

El conocimiento de necesidades de SSR de sus hijos/as adolescentes es más bien indirecto e intuitivo, ya que la comunicación es generalmente escueta y evasiva, siendo pocos los casos que refieren que sus hijos les han comunicado directamente sus dudas, su inicio sexual y/o deseo de prevenir el embarazo. La principal preocupación de ellas es el embarazo precoz, en particular con las hijas. Con los hijos, el temor a las infecciones de transmisión sexual (ITS) ocupa un primer lugar. Estas preocupaciones emergen cuando descubren, o los hijos/as les cuentan, que han iniciado relaciones sexuales (RS) o ha ocurrido el embarazo. Las madres con experiencias personales de embarazo adolescente respaldan la disponibilidad de servicios cuando emergen las inquietudes sexuales y relaciones de pareja de sus hijos. Proponen que deberían buscar en APS información y anticonceptivos, idealmente antes de iniciar RS. Asimismo, están de acuerdo en que consulten en esos servicios por iniciativa propia, solos/as o con sus parejas, sin necesidad de comunicarlo a sus padres si así lo desean. Prefieren que accedan a métodos preventivos antes 
Salud sexual y reproductiva adolescente: visión de los padres - M. X. Luengo-Charath et al

que sufrir consecuencias irreversibles, como un embarazo. En contraste, las madres sin experiencia de embarazo adolescente condicionan y son reticentes, si la atención en SSR incorpora la entrega de anticonceptivos. La aceptación aumenta si se limita la atención al rol orientador educativo, predominando los valores de pareja única y de abstinencia. Prevalece la importancia que atribuyen como prevención, a la dedicación y vigilancia parental de hijos/as. La atención de salud les hace temer que se incentive el inicio de RS, transformándose en barrera para acciones preventivas.

Las madres estiman que los y las adolescentes deberían enfrentar con co-responsabilidad las RS, la prevención del embarazo y las ITS. Se reconoce que las adolescentes tienden a consultar más porque reciben las consecuencias directas del embarazo y por requerir prescripción de anticonceptivos, a diferencia del hombre quien puede comprar preservativos.

La confidencialidad en la atención adolescente, genera aceptación en la gran mayoría y reconocen que esto facilitaría que los/as adolescentes se expresaran más libremente y se sintieran más considerados.

\section{Fase cualitativa: padres}

Preocupados y conscientes de las inquietudes sexuales de sus hijas/os, avalan que consulten acompañados de sus madres. El día a día, la comu- nicación, confianza y acogida, se perciben como responsabilidad materna, siendo ella, quien debe entrar en lo privado o íntimo.

Desde niños han internalizado la sexualidad como socialmente tabú. Se sienten confundidos y sobrepasados, sin manejar la comunicación y orientación en SSR con hijas/os adolescentes. El principal mensaje que les entregan es "cuídense". Para algunos, el inicio de RS está directamente vinculado a la maternidad como consecuencia, $y$ desean que las posterguen hasta finalizada su escolaridad. Por otra parte, consideran que el riesgo de embarazo constituiría un freno natural a iniciarlas.

\section{Fase cuantitativa}

El perfil socio-demográfico de las madres y la distribución según edad y sexo de sus hijos/as, se presenta en la Tabla 1. La proporción de madres que afirmó saber que sus adolescentes mostraban inquietud en relación a la sexualidad, fue de 77,6\% (IC 95\% 74,6-88,6). Las necesidades más importantes de SSR adolescente percibidas fueron: orientación sobre cómo prevenir embarazo y solicitar métodos anticonceptivos; y prevención de ITS-VIH/Sida. Tanto en temas de interés como en percepción de necesidades, se observó una leve tendencia a relacionar el embarazo con las mujeres y las ITS con los varones (Figuras 1 y 2 ).

Casi dos tercios de las madres $(60,8 \%)$ opinaron que los adolescentes se preocupan de averiguar

Tabla 1. Fase cuantitativa: perfil madres encuestadas $(n=125)$

\begin{tabular}{|c|c|c|c|c|c|c|c|}
\hline Edad (en años) & $25-29$ & $30-34$ & $35-40$ & $41-44$ & $45-50$ & $>50$ & Total \\
\hline$\%$ & 4,8 & 20,0 & 27,2 & 21,6 & 20,0 & 6,4 & 100,0 \\
\hline $\begin{array}{c}\text { Nivel } \\
\text { educación }\end{array}$ & $\begin{array}{c}\text { Básica } \\
\text { incompleta }\end{array}$ & $\begin{array}{l}\text { Básica } \\
\text { completa }\end{array}$ & $\begin{array}{c}\text { Media } \\
\text { incompleta }\end{array}$ & \multicolumn{2}{|c|}{$\begin{array}{l}\text { Media } \\
\text { completa }\end{array}$} & $\begin{array}{l}\text { Técnica superior o } \\
\text { universitaria }\end{array}$ & Total \\
\hline$\%$ & 8,8 & 13,6 & 29,6 & \multicolumn{2}{|c|}{40,0} & 8,0 & 100,0 \\
\hline \multicolumn{8}{|c|}{ Distribución porcentual según edad y sexo de los hijos/as adolescentes } \\
\hline Edad & \multicolumn{2}{|c|}{$\begin{array}{l}10 \text { a } 14 \\
(n=56)\end{array}$} & $\begin{array}{c}15 \text { a } 19 \\
(n=39)\end{array}$ & \multicolumn{3}{|c|}{$\begin{array}{l}\text { Hijos de ambos grupos de edades } \\
\qquad(\mathrm{n}=30)\end{array}$} & $\begin{array}{l}\text { Total general } \\
\text { por sexo }\end{array}$ \\
\hline \multicolumn{8}{|l|}{ Sexo } \\
\hline Sólo hombres & \multicolumn{2}{|c|}{51,8} & 48,7 & \multicolumn{3}{|c|}{40,0} & 48,0 \\
\hline Sólo mujeres & \multicolumn{2}{|c|}{39,3} & 48,7 & \multicolumn{3}{|c|}{10,0} & 35,2 \\
\hline Hijos de ambos sexos & \multicolumn{2}{|c|}{8,9} & 2,6 & \multicolumn{3}{|c|}{50,0} & 16,8 \\
\hline Total general & \multicolumn{2}{|c|}{100,0} & 100,0 & \multicolumn{3}{|c|}{100,0} & 100,0 \\
\hline
\end{tabular}



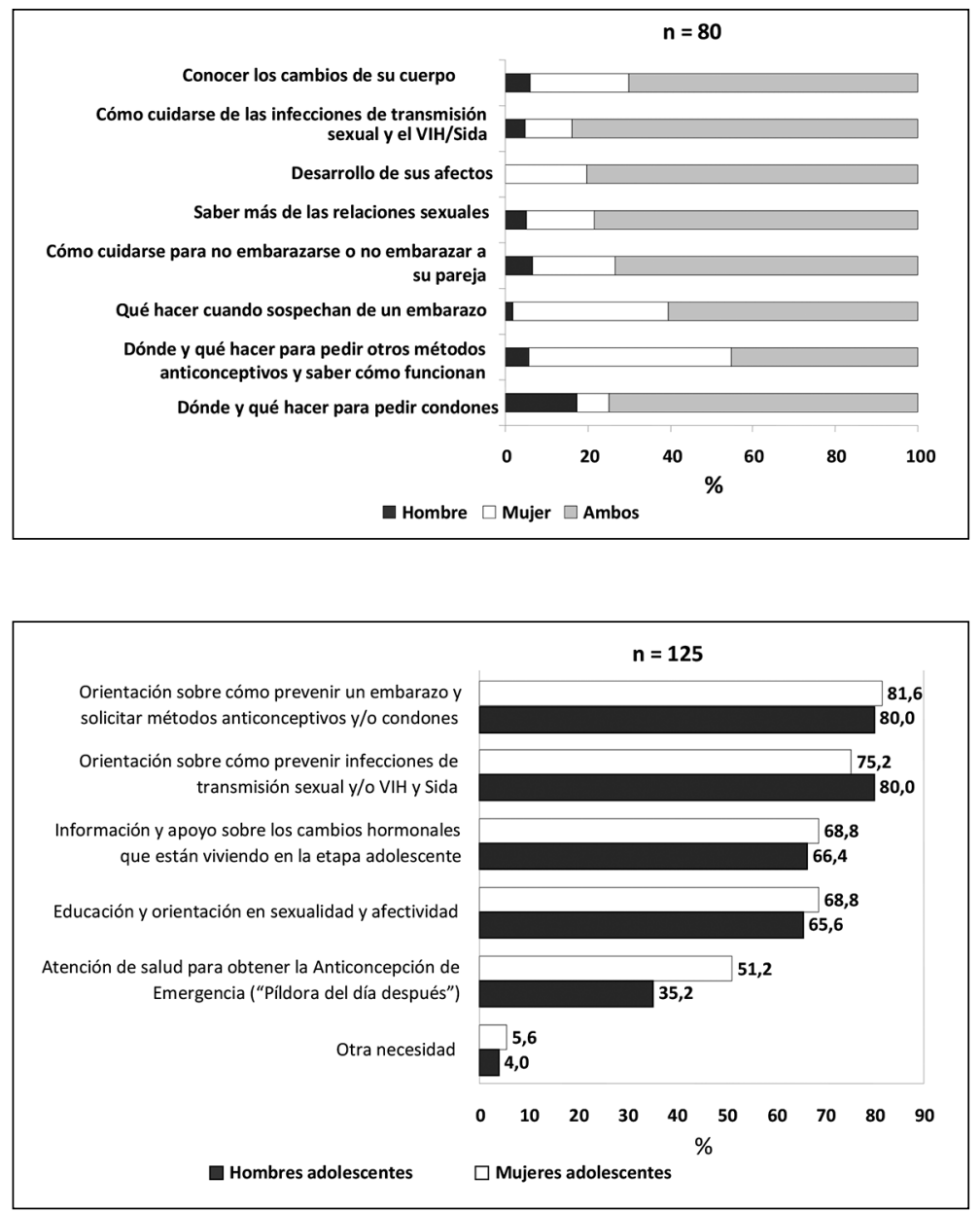

Figura 1. Opinión materna sobre temas de interés en SSR según sexo de los adolescentes.
Figura 2. Percepción materna sobre las principales necesidades adolescentes sobre SSR, según sexo. por sí mismos sobre SSR y creían que la información la obtienen principalmente desde los pares, Internet y a través de los profesores. Estimaron que los padres contribuyen en menor porcentaje (madres en $36,8 \%$; padres $23,7 \%$ ) y, en último lugar, los profesionales de salud de APS $(7,9 \%)$. Sin embargo, independientemente del sexo de los adolescentes, conversarían sobre sexualidad con ambos padres (Tabla 2). La mayoría de las madres manifestó que sus hijos/as habían recibido educación sexual fuera del hogar $(85,6 \%)$, fundamentalmente en el colegio $(94,4 \%)$.

Una alta proporción de madres (76\%) sabía que sus hijos/as podían solicitar atención de SSR en APS, a través de los profesionales, manifestando la gran mayoría $(96,8 \%)$ estar de acuerdo con la posibilidad que los adolescentes puedan consultar, independiente de la edad y sexo de los hijos
(Tabla 3). Los motivos más frecuentes de consulta serían: prevenir embarazo no deseado e ITS $(81,8 \%)$; poder consultar con libertad y confianza sus inquietudes sobre sexualidad $(71,1 \%)$; recibir orientación profesional especializada $(66,1 \%)$ e información sobre los cambios de crecimiento y desarrollo propios de la edad (62,8\%).

El 61\% de las madres estuvo de acuerdo con la confidencialidad en la consulta adolescente, con leve tendencia a mayor aprobación en aquellas que vivieron su propio embarazo adolescente. La experiencia cercana de embarazo adolescente fue del orden de $75,2 \%$ : la propia $39,4 \%$ y $26,6 \%$ con algún hijo/a.

Más de 50\% de las madres opinó que el rango etario adecuado para acercarse a los servicios sería entre 10 y 13 años, previo al inicio de la pubertad, sin diferencias significativas por sexo. Sólo 4 
Salud sexual y reproductiva adolescente: visión de los padres - M. X. Luengo-Charath et al

Tabla 2. Con quiénes conversan en el hogar sobre sexualidad según grupos de edad y sexo de los hijos adolescentes

\begin{tabular}{|c|c|c|c|c|c|c|}
\hline \multirow[t]{2}{*}{$\begin{array}{l}\text { Con quién conversan sobre } \\
\text { sexualidad en el hogar }\end{array}$} & \multicolumn{2}{|c|}{ Sólo hombres } & \multicolumn{2}{|c|}{$\begin{array}{l}\text { Sexo hijos adolescentes } \\
\text { Sólo mujeres }\end{array}$} & \multicolumn{2}{|c|}{ Ambos sexos } \\
\hline & $\mathbf{n}$ & $\%$ & $\mathbf{n}$ & $\%$ & $\mathbf{n}$ & $\%$ \\
\hline Sólo con la mamá & 18 & 34,0 & 17 & 43,6 & 8 & 40,0 \\
\hline Sólo con el papa & 0 & 0,0 & 0 & 0,0 & 1 & 5,0 \\
\hline Con ambos, mamá y papá & 35 & 66,0 & 22 & 56,4 & 11 & 55,0 \\
\hline Total & 53 & 100,0 & 39 & 100,0 & 20 & 100,0 \\
\hline
\end{tabular}

Tabla 3. Grado de acuerdo de las madres con la consulta por atención en SSR según sexo y edad de sus hijos adolescentes

\begin{tabular}{|c|c|c|c|c|c|c|}
\hline Grado de acuerdo & $\begin{array}{c}\text { Sólo } \\
\text { hombres } \\
\% \\
(n=60)\end{array}$ & $\begin{array}{c}\text { Sexo } \\
\text { Sólo } \\
\text { mujeres } \\
\% \\
(n=44)\end{array}$ & $\begin{array}{c}\text { Ambos } \\
\text { sexos } \\
\% \\
(n=21)\end{array}$ & $\begin{array}{c}10 \text { a } 14 \\
\% \\
(n=56)\end{array}$ & $\begin{array}{c}\text { Edades } \\
15 \text { a } 19 \\
\% \\
(n=39)\end{array}$ & $\begin{array}{c}\text { Ambos grupos } \\
\text { de edad } \\
\% \\
(n=30)\end{array}$ \\
\hline Muy de acuerdo & 85,0 & 72,7 & 85,6 & 76,8 & 79,5 & 90,0 \\
\hline De acuerdo & 13,3 & 25,0 & 4,8 & 17,8 & 17,9 & 10,0 \\
\hline En desacuerdo & 1,7 & 2,3 & 4,8 & 3,6 & 2,6 & 0,0 \\
\hline Muy en desacuerdo & 0,0 & 0,0 & 4,8 & 1,8 & 0,0 & 0,0 \\
\hline
\end{tabular}

madres señalaron su desacuerdo con la consulta, temiendo a autorizarlos a iniciar actividad sexual, incentivarlos u otorgarles mayor libertad sexual.

Un alto porcentaje de madres señaló estar de acuerdo con que sus hijos consultaran si estuvieran decididos a iniciar RS $(84,8 \%)$ y sobre $50 \%$ si estuvieran comprometidos en una relación afectiva seria y permanente en el tiempo, o si requirieran atención de necesidades específicas de SSR como: controlar un embarazo, tratar una ITS o solicitar anticonceptivos, incluida la anticoncepción de emergencia.

Las madres que habían solicitado atención de SSR con sus hijos/as en APS, la evaluaron positivamente.

Sobre $90 \%$ de las madres que aprobaban la consulta, identificó como importantes los siguientes atributos de los servicios de SSR para adolescentes: que sean gratis; que quienes los atienden se preocupen por sus problemas; que les sirvan para evitar embarazo; que no les dieran vergüenza ir a consultar; y de fácil acceso. Condiciones del profesional que brinda la atención serían: trato acogedor, cálido y que transmita confianza; capacitado/a y/o especialista en jóvenes, que sepa escuchar e informar según las necesidades y estilos adolescentes. Como características del lugar físico: ambiente agradable y juvenil, privacidad y con material educativo pertinente.

Finalmente, consultadas sus expectativas respecto al enfoque de un programa de atención en SSR, más de dos tercios de ellas señalaron que esté orientado a prevenir el embarazo, las ITS y el VIH/sida (Tabla 4).

\section{Discusión}

Se confirma que el interlocutor válido de los/ as adolescentes para conversar sobre sexualidad es la madre, consistente con estudios nacionales que la reconocen como referente más importante en este tema ${ }^{10,19,20}$.

Si bien se confirma que un alto porcentaje las madres conocían las inquietudes sobre sexualidad y las necesidades de SSR de sus hijos/as, existe discrepancia en la forma en que ellas reportan cómo 
Salud sexual y reproductiva adolescente: visión de los padres - M. X. Luengo-Charath et al

Tabla 4. Enfoque esperado de un programa que otorga servicios de SSR para adolescentes según opinión de las madres $(n=121)$

\begin{tabular}{lc}
\hline Enfoque & $\mathbf{\%}$ \\
\hline Orientado a prevenir el embarazo y las Infecciones de Transmisión Sexual, el VIH y SIDA & 66,1 \\
\hline Alternativa de apoyo a los padres para la educación en la salud sexual y reproductiva de sus hijos & 47,9 \\
\hline Integral, que aborde todos los aspectos que involucran el desarrollo adolescente (bio-psico-social) & 47,1 \\
\hline Permita tomar conciencia de sus responsabilidades & 33,1 \\
\hline Participativo, que promueva el protagonismo del adolescente (que los jóvenes puedan opinar) & 25,5 \\
Contemple orientación para los padres con sus hijos adolescentes ("como se lleva la edad") & 21,5 \\
Educativo y de orientación, más que de tratamiento médico & 17,4 \\
Más allá de la "consejería" que les entreguen los métodos anticonceptivos & 15,7 \\
Que sea un programa que se preocupe principalmente de los problemas de salud física & 10,7
\end{tabular}

se informan de éstas. Según las entrevistadas, más bien lo intuyen y perciben indirectamente, sin embargo, en la encuesta manifiestan que se informan directamente a través de sus hijos/as.

Reconocen que sus hijos buscan información sobre SSR de manera independiente, siendo sus pares la mayor fuente de información, observándose una brecha con referentes adultos como padres y profesores.

La mayoría de las madres manifestó que sus hijos habían recibido educación sexual fuera del hogar, rol protagónico del sector educación avalado por la política de educación para la sexualidad, con garantía y derecho universal, a pesar de que aún su implementación no ha logrado consolidación y cobertura universal ${ }^{21-24}$.

Los profesionales de APS son mencionados en último lugar como fuente de información en SSR. Esto puede tener varias explicaciones, más allá de las conocidas barreras de acceso a servicios ${ }^{11,12}$. Siendo la sexualidad inherente al ser humano y la reproducción eventos de una vida saludable, parece lógico que sólo se genere la consulta cuando aparece un problema de salud o para iniciar control prenatal.

Intervenciones como la Consejería recientemente sistematizada por el Ministerio de Salud, constituyen estrategias de promoción de salud integral. Su objetivo, al parecer escasamente difundido entre los padres, es acompañar hacia un desarrollo integral armónico y saludable, inserto en el contexto valórico familiar, favoreciendo la comunicación efectiva padres-hijos y esperando un impacto positivo en la salud y calidad de vida futuras ${ }^{25,26}$.

Hubo una sólida consistencia entre información requerida, percepción de necesidades y motivos de consulta de SSR adolescente, declaradas por las madres. Prevenir el embarazo, las ITS y/o VIH/Sida, y recibir información y apoyo, fueron temas prioritarios. Los resultados coinciden con los intereses de los/as adolescentes recogidos en un estudio previo. Esto constituye una fuerte evidencia que avala la necesidad de promoción y prevención en SSR para el grupo etario adolescente ${ }^{10}$.

Interesante desde una perspectiva de género fue observar una tendencia en el discurso de madres a promover la co-responsabilidad entre los $y$ las adolescentes en estos temas, revelando un avance hacia una igualdad de género.

Si bien la mayoría de las madres sabía de la atención de SSR para adolescentes en APS, un pequeño porcentaje manifestó haber consultado con sus hijos, fundamentalmente mujeres, confirmando barreras de acceso a ésta.

Disminuir el embarazo adolescente es un gran desafío y una meta sanitaria comprometida por el país, por lo que estrategias de prevención resultan prioritarias. En Chile los nacimientos en madres adolescentes muestran tendencia a disminuir en forma global, mientras en menores de quince años grupo que concentra los mayores riesgos de morbi-mortalidad perinatal, las cifras permanecen relativamente estables a pesar de los esfuerzos de políticas y programas ${ }^{27,28}$.

Casi dos tercios de las madres aprobaron la confidencialidad. Este es un aspecto fundamental de la atención ya que las dudas respecto de 
Salud sexual y reproductiva adolescente: visión de los padres - M. X. Luengo-Charath et al

su resguardo aleja en especial a adolescentes en riesgo, afectando particularmente a mujeres con requerimientos en el área de la $\mathrm{SSR}^{29,30}$. Hallazgo interesante que confirma lo que indica la ley que fija normas sobre información, orientación y prestaciones en materia de regulación de la fertilidad. Un tercio de las madres estaba en desacuerdo con respetar la confidencialidad, por temor a estimular la actividad sexual de sus hijos/as, hecho que requiere ser abordado con nuevos estudios.

Las características más importantes de los servicios de SSR para adolescentes señaladas por las madres, coinciden con las recomendaciones técnicas referentes a servicios amigables ${ }^{4,5,8,31}$. Los principales atributos valorados fueron: la gratuidad de la atención; la especialización de los equipos profesionales en el tema de SSR de adolescentes; la disposición a brindar apoyo a padres y madres para comprender mejor a sus hijos y manejar con mayor habilidad inquietudes y necesidades de su desarrollo sexual; la privacidad y confidencialidad de la consulta y; el respeto a los valores familiares.

De lo expuesto, es posible concluir que para optimizar el uso de los servicios de SSR para adolescentes y alcanzar un impacto preventivo, no sólo se hace necesaria una atención acorde a sus expectativas y necesidades, si no también articulada con las de padres y madres. Se precisa que las aprensiones de madres y padres sean consideradas por los equipos de salud y realicen una labor de información, acercamiento y sensibilización respecto del beneficio de las actividades de promoción y prevención. Esto contribuiría a romper prejuicios y cambiar creencias erróneas que funcionan actualmente como frenos y barreras para que los adolescentes consulten.

Finalmente, los resultados ilustran el respaldo de padres y madres urbanos, beneficiarios del Sistema Público de Salud, a la oferta de servicios de SSR para adolescentes en APS. Esperan una acción colaborativa de éstos en las actividades de formación, orientación y prevención, particularmente del embarazo, ITS y VIH/Sida.

Políticas que favorezcan el acceso a una óptima atención de SSR y la difusión sobre la existencia de servicios preventivos, constituyen respuesta a demandas de los padres y una contribución al pleno y saludable desarrollo humano adolescente ${ }^{32}$.

Agradecimientos: A la Dra. Adela Montero, por su aportes a la encuesta en calidad de juicio de experto y a las autoridades de los Centros de Salud de Atención Primaria y de Atención Especializada para Adolescentes donde se realizó este estudio. Especialmente a las madres y padres de adolescentes, quienes anónima y desinteresadamente colaboraron con la investigación. Al Instituto Chileno de Medicina Reproductiva-ICMER, que otorgó el respaldo y apoyo para la realización de este estudio.

\section{Referencias}

1. Gobierno de Chile, Ministerio de Salud, OPS/KELLOGG. Luengo X. 1994; La Salud del Adolescente y Joven en Chile y los Servicios de Salud. Informe del Proyecto Apoyo a las Iniciativas Nacionales. Chile.

2. Gobierno de Chile, Ministerio de Salud. 1999; Política Nacional de Salud para Adolescentes y Jóvenes en Chile.

3. Gobierno de Chile, Ministerio de Salud. 2008; Política Nacional de Salud para Adolescentes y Jóvenes en Chile 2008-2011.

4. OPS/OMS/UNFPA. 2000; Recomendaciones para la atención integral de salud de los y las adolescentes, con énfasis en salud sexual y reproductiva. Washington D.C.

5. OPS/OMS. 2005; IMAN Servicios: Normas de atención de salud sexual y reproductiva. Washington D.C.

6. UNFPA/ICMER. 2005; Capacitación en Derechos y Salud Sexual y Reproductiva de Adolescentes en América Latina y El Caribe.

7. World Health Organization (2012). Department of Maternal, Newborn, Child and Adolescent Health. Making health services adolescent friendly. Developing national quality standards for adolescent friendly health services.

8. Gobierno de Chile, MINSAL. 2004; Atención amigable para adolescentes en establecimientos de atención primaria. Orientaciones técnicas. Disponible en: http://207.58.191.15:8180/xmlui/bitstream/handle/1234 56789/151/23_SALUD_ATENCION_PRIMARIA.pdf? sequence $=1$ [Consultado el 18/12/2013].

9. Senderowitz J. 1999. Logrando Servicios de Salud Reproductiva «Amigables» para Adolescentes. Focus and Young adults. Disponible en: www.pathfind.org/pf/ pubs/focus/RPPS-Papers/spmaking.pdf [Consultado el 18/12/2013].

10. Luengo Charath MX, Millán-Klüsse T, Zepeda Ortega AJ, Tijero Méndez M. Adolescentes estudiantes urbanos: conocimientos sobre la atención de Salud Sexual y Reproductiva en el Sistema Público de Salud. Rev Chil Pediatr 2012; 83 (6): 540-51.

11. Convenio Agencia Española de Cooperación Internacional para el Desarrollo (AECID), Fondo de Población 
Salud sexual y reproductiva adolescente: visión de los padres - M. X. Luengo-Charath et al

de las Naciones Unidas (UNFPA)-Oficina Regional para América Latina y el Caribe, Comité Regional Andino para la Prevención del Embarazo Adolescente (2010). Barreras para el acceso de adolescentes y jóvenes a servicios de salud. Propuesta para su identificación y superación. Documento regional. Disponible en: http://www. unfpa.org.co/uploadUNFPA/file/BarrerasJovenesWeb. pdf [Consultado el 13/12/2013].

12. Gobierno de Chile, Ministerio de Salud, Programa Nacional de Salud Integral de Adolescentes y JóvenesCultura Salud (2011). Estudio de barreras y acceso a los Servicios de Salud para la prevención del embarazo adolescente en Chile. pp.104.

13. Boonstra DH, Young People Need Help in Preventing Pregnancy and HIV; How Will the World Respond? Guttmacher Policy Review. Summer 2007; 10 (3): 2-8.

14. Churchill D, Allen J, Pringle M, Hippisley-Cox J, Ebdon $\mathrm{D}$, Macpherson $\mathrm{M}$, et al. Consultation patterns and provision of conception in general practice before teenage pregnancy: case-control study. BMJ 2000; 321 (7259): 486-9.

15. Organización Panamericana de la Salud (2010) "Plan y Estrategia Regional de Salud Adolescente". Washington DC. Disponible en: http://new.paho.org/hq/dmdocuments/2011/Estrategia-y-Plan-de-Accion-Regionalsobre-los-Adolescentes-y-Jovenes.pdf [Consultado el 18/12/2013].

16. Luengo X. Necesidades de Salud. En: Molina R, Sandoval J, González E, Editores. Salud Sexual y Reproductiva en la Adolescencia. Santiago, Chile: Editorial Mediterráneo Ltda.; 2003. pp. 102-10.

17. Bearinger L, Sieving R, Ferguson J, Sharma V. Adolescent health 2: Global perspectives on the sexual and reproductive health of adolescents: patterns, prevention, and potential. The Lancet 2007; 369: 1220-31.

18. Ministerio de Desarrollo Social, Gobierno de Chile. Objetivos de Desarrollo del Milenio, Objetivo 5. Indicadores adicionales ODM. Disponible en: http://siis. ministeriodesarrollosocial.gob.cl/indicadoresmilenio/ [Consultado el 26/11/2013].

19. Ministerio de Educación-MINEDUC, Gobierno de Chile y ADIMARK 2004. Resultados del estudio educación en sexualidad. Santiago de Chile.

20. Fernández FL, Bustos ML, González WL, Palma AD, Villagrán AJ, Muñoz NS. Creencias, actitudes y conocimientos en educación sexual. Rev Med Chile 2000; 128 (6): 574-83.

21. Ministerio de Educación, Gobierno de Chile. 1993. Política de Educación en Sexualidad.

22. Ministerio de Educación, Gobierno de Chile. 2006. Plan de Educación en Sexualidad y Afectividad.
23. Ley 20.418 (28.01.2010) Fija Normas sobre información, Orientación y Prestaciones en materia de regulación de la Fertilidad. Disponible en http://www.bcn.cl/buscador_beta/buscador_rpta?busqueda $=$ ley +20.418 \&pagin $\mathrm{a}=1$ \&mindate $=+\&$ maxdate $=+\&$ sor $\mathrm{t}=+\&$ fuente $=$ PCiu dadano\%2BPLegislativo\%2BAsiaPacifico\%2BCatalog $\mathrm{o} \& \mathrm{~b}=1$ \&imageField. $\mathrm{x}=0$ \&imageField. $\mathrm{y}=0$ [Consultado el 26/11/2013].

24. UNFPA Abogacía ICPD+15 CHI1R11A y Universidad Central de Chile. Seguimiento a la Ley 20.418: Prevención del embarazo adolescente, educación sexual y anticoncepción de emergencia. Santiago, Chile, 2011. Serie de Documentos No 1. http://www.icmer. org/documentos/adolescencia/te-educa-sexual-y-aechile\%20AMConcha\%2029.04.2013.pdf [Consultado el 26/11/2013].

25. Ministerio de Salud de Chile. Guía práctica de Consejería para Adolescentes y Jóvenes. Orientaciones Generales. Dirigida a los equipos de Atención Primaria, 2011. Disponible en: http://web.minsal.cl/portal/url/item/ aaa27720f365a745e04001011e011120.pdf [Consultado el 29 de noviembre de 2013].

26. Millán T, Morera I, Vargas N. Consejería a adolescentes: descripción epidemiológica y motivos de consulta. Rev Med Chile 2007; 135: 457-63.

27. Ministerio de Salud. Gobierno de Chile. Situación actual del embarazo adolescente en Chile. Julio de 2013. Disponible en: http://www.minsal.cl/portal/url/item/ c908a2010f2e7dafe040010164010db3.pdf [Consultado el 26/08/2013].

28. Federación Latino Americana de Sociedades de Obstetricia y Ginecología-FLASOG, Comité de Derechos Sexuales y Reproductivos. Factores relacionados con el embarazo y la maternidad en menores de 15 años en América Latina y El Caribe. Lima, Perú, 2011.

29. Lehrer J, Pantell R, Tebb K, Shafer MA. Forgone Health Care among U.S. Adolescents: Associations between Risk Characteristics and Confidentiality. Concern Journal of Adolescent Health 2007; 40 (3): 218-26.

30. Society for Adolescent Medicine. Confidential Health Care for Adolescents: Position Paper. J Adolesc Health 2004; 35: 1-8.

31. WHO/Department of Maternal, Newborn, Child and Adolescent Health. Making health services adolescent friendly. Developing national quality standards for adolescent friendly health services. Geneva, Switzerland, 2012.

32. Maddaleno M, Morello P, Infante-Espínola F. Salud y desarrollo de adolescentes y jóvenes en Latinoamérica y el Caribe: desafíos para la próxima década. Salud Pública Mex 2003; 45 (supl 1): S132-9. 